\title{
LA ENSEÑANZA DEL ARTE ELECTRÓNICO MEXICANO: ALTERNATIVAS PARA ESCENARIOS LOCALES
}

\section{Cynthia Patricia Villagómez Oviedo}

Universidad de Guanajuato (México). Dpto. de Diseño

\section{Resumen}

La enseñanza del arte electrónico mexicano es la puerta de entrada a la comprensión de los distintos recursos científicos y tecnológicos, así como la entrada a redes o comunidades de desarrollo de conocimientos en línea, las cuales cubren la necesidad de obtener un conocimiento específico. El arte electrónico puede hacer posible la entrada a estos recursos, entre otros, necesarios para el avance de un grupo social o de la sociedad en conjunto. La educación universitaria en países en vías de desarrollo está supeditada a estándares nacionales e internacionales arcaicos en la implementación de rediseños o para la creación de nuevos programas de estudio, lo que obstaculiza la introducción de nuevas carreras del área artística, cuya base es la flexibilidad para la selección de contenidos, debido al cambio constante que experimentan las diferentes corrientes artísticas, verbigracia, la reutilización de recursos y materiales en el arte electrónico. Este artículo reflexiona sobre las desventajas de las guías institucionales para la creación de programas educativos y las ventajas de implementar nuevas normas que contribuyan a la flexibilidad de los planes de estudio.

\section{Palabras clave: ENSEÑANZA ARTÍSTICA UNIVERSITARIA (MEXICO); PROGRAMAS EDUCATIVOS (MEXICO); ARTE ELECTRÓ- NICO (MEXICO); REUTILIZACIÓN}

\section{THE TEACHING OF MEXICAN ELECTRONIC ART. ALTERNATIVES FOR LOCAL CONDITIONS}

\section{Abstract}

The teaching of Mexican Electronic Art facilitates the comprehension of the scientific and technological knowledge. Through Electronic Art people can reach online communities, being capable to solve specific problems regarding their artistic developments or just to know more about science and technology. Electronic Art can make major improvements on a specific group or an entire society. The professional education at the universities in countries with economic adversities depends on national and international standards, most of them archaic, but very important if a university wants to create or redesign undergraduate programs, these standards are an obstacle for art programs, because, those needs flexibility to choose diverse class subjects that might belong to other educative programs. This article talked about disadvantages of institutional documents for the creation of educational programs and the advantages of apply new forms to create art programs that could contribute to a flexible educational programs.

Keywords: UNIVERSITY ARTISTIC LEARNING (MEXICO); EDUCATIONAL PROGRAMS (MEXICO); ELECTRONIC ART (MEXICO); REUSE

\footnotetext{
Villagómez Oviedo, Cynthia Patricia. 2020. "La enseñanza del arte electrónico mexicano: Alternativas para escenarios locales". AusArt 8 (1): 103-115. D0I: 10.1387/ausart.21551
}

\section{AUSART}




\section{SOBRE LA RELACIÓN ARTE, CIENCIA Y TECNOLOGÍA}

Es necesario dar inicio a la reflexión en torno a la educación universitaria de las artes, definiendo el campo que nos ocupa, a saber, el arte electrónico o el arte con el uso de tecnología, el cual, combina en gran medida arte, ciencia y tecnología. "¿No recuerda esto a la época del Renacimiento, en la que Leonardo da Vinci, además de artista, era inventor, Miguel Ángel, ingeniero, y Galileo Galilei, por contra, también artista?" (Lieser 2010, 12). Combinaciones que ofrecen ventajas, ya que se traducen en nuevas posibilidades que ampliaron los horizontes del arte. Por otra parte, uno de los fundamentos del desarrollo creativo se encuentra en la combinación entre áreas que suponemos no habrían de tocarse, el arte electrónico combina varias áreas aparentemente disímbolas, lo que lo convierte en un caldo de cultivo propicio para nuevas ideas, como también en un área que precisa cambios constantes, así como un abordaje innovador en los planes de estudio en la educación universitaria. A tenor de lo citado podemos mencionar que, el arte electrónico es una rama del arte que utiliza los medios y herramientas de la electrónica análoga o digital, que consecuentemente definen la época en la que vivimos. De tal manera que esta mezcla de recursos genera un ámbito más amplio de trabajo en la creación y educación artística, que ofrece tanto estímulos interesantes como nuevos retos en la clasificación y su definición.

\section{FORMACIÓN DE ARTISTAS ELECTRÓNICOS EN UNIVERSIDADES MEXICANAS}

No es sino hasta finales del siglo $\mathrm{XX}$ e inicios del siglo $\mathrm{XXI}$, que las universidades mexicanas vieron la formación de artistas electrónicos como una posibilidad. Antes de eso, los antecedentes del arte y la tecnología, se encuentran en las artes aplicadas, cuando en la década de los noventa se introdujeron los primeros ordenadores. Casos como el de la Escuela de Diseño del Instituto Nacional de Bellas Artes en la Ciudad de México, que a inicios de esa década adquirió cinco ordenadores de la marca Apple Macintosh para los alumnos de la carrera en diseño gráfico de los últimos semestres y el caso de la Universidad Iberoamericana (de educación privada), quienes fundan la Licenciatura en Diseño Gráfico en 1968 y no es sino hasta 1984 con la comercialización del primer ordenador Apple, que los introdujeron en esa institución. 
Por lo que la experimentación artística tecnológica en la educación superior en el caso mexicano, nació en las escuelas de diseño, es decir, en las escuelas de artes aplicadas. De ahí que, incluso, artistas tecnológicos o electrónicos mexicanos tengan estudios inconclusos en diseño gráfico, tales como, Arcángel Constantini y Leslie García, o quienes tienen formación terminada como diseñadores, como es el caso de Iván Abreu.

Actualmente universidades nacionales cuentan con programas en arte digital, donde forman artistas electrónicos, la mayoría son instituciones privadas, como el Tecnológico de Monterrey, UNITEC, Universidad del Valle de México y Centro en la Ciudad de México. En el caso de las universidades públicas que forman artistas electrónicos están, la Universidad de Guanajuato, la Universidad Autónoma del Estado de México, la Universidad Autónoma Metropolitana, entre otras. Pese a lo anterior, es muy importante destacar que en muchos casos los egresados se dedican profesionalmente al desarrollo de proyectos de diseño (gráfico, web ${ }^{1}$, etc.), y no producen desarrollos artísticos, sino proyectos de diseño, que son más redituables.

\section{CURRÍCULOS FLEXIBLES CONTRA POLÍTICAS DE AUSTERIDAD}

Hoy en día una de las preocupaciones de quienes han estado o estamos en los equipos de trabajo de rediseño o creación de nuevas carreras en el área de artes es, lo complejo que resulta renovar programas de licenciatura existentes, como los de diseño o de arte digital, de manera que puedan responder rápidamente a la vorágine de cambios científicos y tecnológicos actuales, y por otra parte, que constituyan un corpus de conocimientos lo más completo posible para que a través del proceso de adquisición de los mismos, el estudiante egrese con el desarrollo de las competencias necesarias que le posibiliten su pervivencia, en este caso, como artista electrónico.

De ahí que el problema sea cómo dar contenidos de vanguardia, los que lógicamente cambian constantemente y tener currículos flexibles adaptados a los estudiantes de hoy, muchos de ellos que estudian y trabajan, son padres de familia, rebasan la edad promedio de un alumno universitario, entre otras características, con políticas de rediseño y creación de nuevas carreras encorsetados en guías de diseño curricular ajustadas al know how del siglo pasado, 
es decir, cuando se ajustan los currículos, éstos se hacen de la única manera que se conoce, y ésta es: como se hacía antes. Por lo que se trabaja con guías de mejoras curriculares anticuadas, que por una parte preconizan la necesidad de contar con currículos flexibles (Universidad de Guanajuato 2016, 85), y por otra, se enfrentan a una realidad operativa que contraviene sus preceptos; además de que no favorecen el intercambio de conocimientos entre disciplinas, luego entonces, áreas como el Arte tecnológico donde la interdisciplina es fundamental, se ven obstaculizadas en su implementación y desarrollo.

Ejemplo de lo anterior, es cuando se quiere trabajar con profesores investigadores de otras áreas, en estos casos, en ocasiones se aplican solicitudes para que los proyectos tengan financiamiento externo a las propias universidades, este financiamiento se otorga a través de la formación de equipos llamados Cuerpos Académicos (CA), los cuales deben de constituirse formalmente dentro de las universidades y son registrados ante instancias federales. Pero, dentro de las universidades es difícil que se permita a los profesores investigadores formar estas células llamadas Cuerpos Académicos con pares de otras áreas y sedes. Ante tal situación, el personal administrativo argumenta que es debido a que no sabrían en qué campus dejar los recursos materiales (equipos) obtenidos por parte del financiamiento otorgado; además de otras cuestiones políticas, como que se otorgan recursos en ciertos rubros, de acuerdo al número de Cuerpos Académicos que tenga cada campus. Luego entonces, pensar en la colaboración entre artistas, matemáticos, biólogos, físicos, entre otros científicos -en estas condiciones-, podría desalentar a cualquiera o, por el contrario, puede convertirse en un motivo importante para los profesores investigadores dedicados al arte electrónico e incluso para alumnos que deseen cursar la mayor parte de sus asignaturas en distintas facultades, pugnen por nuevas regulaciones que faciliten el intercambio entre áreas.

Aunado a lo anterior, la educación universitaria del arte electrónico tiene aspectos fundamentales, como son, el trabajo en equipos multidisciplinarios con profesionales de distintas áreas, tales como ingeniería electrónica, filosofía de la ciencia, física, química, biología, robótica, entre muchos otros, esto significa que, dependiendo del proyecto, el equipo de profesionales cambia. Por lo que los alumnos que desean cursar sus asignaturas en diferentes facultades enfrentan candados dentro de la planeación de cada carrera difíciles de sortear, como lo es que los sistemas educativos solo validan cierto porcentaje de asignaturas para unas y otras áreas (generalmente el $20 \%$ de la currícula es asignada al área general, que son materias que pueden ser cursadas por alumnos de otras carreras). En consecuencia, si se desea que una institución 
tenga Programas Educativos que formen artistas electrónicos, la recomendación sería tener un plan de estudios sumamente flexible que permita tanto a sus alumnos y profesores cursar e impartir asignaturas en otras facultades dependiendo de sus intereses, así como de los proyectos profesionales y de investigación que se realicen, donde el desarrollo de la competencia de trabajo en grupos colaborativos sea fundamental.

Por lo que se advierte que la realidad contradice lo que se ha establecido como norma, por citar un ejemplo, la Guía para la modificación de programas educativos de licenciatura de la Universidad de Guanajuato (2016), en el apartado Flexibilidad Académica establece que la universidad promueve:

un modelo organizativo más abierto, dinámico y polivalentequepermite transformar las estructuras organizativas académicas mediadas por la interdisciplinariedad y el trabajo integrado, deacuerdo con el artículo 4 del Estatuto Orgánico, el cual a la letra establece: El nivel superior se organizará en Campus, Divisiones y Departamentos. Los mecanismos y procedimientos que se diseñen para el funcionamiento de los Campus, deberán atender a los criterios de calidad e identidad institucional, vigilando que se garantice la integración de la comunidad académica y su vinculación con la sociedad. Esta flexibilidad, fortalece las interrelaciones de unas unidades con otras, promoviendo la generación de formas de trabajo más socializado, participativo y cooperativo, coherente con las formas de organización del conocimiento establecido en cada PE [Programa Educativo].

En suma, ¿Cómo se puede trabajar con estudiantes y profesores de otras áreas, si las estructuras administrativas y los propios límites de los Programas Educativos no lo permiten? Por lo que, si se quiere una educación que haga posible la introducción de disciplinas artísticas actuales en las universidades, se deberá hacer transformaciones coyunturales en la educación universitaria, no solo cambios contemplados en papeles y documentos, sino cambios reales que contribuyan a una sociedad más incluyente y por tanto más universal.

De lo anterior se desprende y se puede aplicar a los países que no pertenecen a las primeras potencias económicas mundiales, tales como México y en general a los países latinoamericanos, en donde las universidades tienen planes muy estrictos de austeridad (Universidad de Guanajuato 2018), de tal manera que ante la situación de escasez de recursos materiales, de personal académico y administrativo, se deben actualizar los programas de carrera con el mismo equipo material y humano, es decir, con los mismos profesores, (lo 
que llaman desarrollo de Programas Educativos con planta instalada), de ahí surge el cuestionamiento: ¿Cómo impartir Arte tecnológico o electrónico con una planta docente que no fue formada en esa área? O más complejo aún ¿Cómo abrir nuevos programas de Licenciatura como lo es arte electrónico o digital si no se cuenta suficientes recursos?

\section{LA REUTILIZACIÓN Y LA TECNOLOGÍA OBSOLETA COMO ALTERNATIVAS}

Tras más de una década estudiando los procesos de producción de artistas electrónicos mexicanos (Villagómez 2017), hemos observado que, ni la escasez de recursos materiales, ni las limitaciones en conocimientos sobre algún tema, han sido obstáculos para que los artistas electrónicos produzcan obra. En la actualidad, la diferencia la aportan las redes o comunidades de conocimiento en línea, el uso de materiales de deshecho y la tecnología obsoleta.

De tal manera que el estudio del arte electrónico y sus ramas son un ámbito complejo, en parte porque las obras producidas han contribuido a señalar la existencia de determinados problemas: científicos, ambientales, sociales, culturales, entre otros. Es debido a la naturaleza de los temas, que los desarrollos artísticos han sido abordados haciendo uso de los conocimientos de diversas disciplinas, es decir, se involucran distintas perspectivas no sólo la artística. Ejemplos de lo anterior son: Nanodrizas (2009) de Arcángel Constantini, hace uso de la bioremediación, la robótica, el arte sonoro, entre otros; Alzado vectorial (2000) de Rafael Lozano-Hemmer, utiliza la robótica, el diseño de software, las redes e Internet, la óptica, entre otros; Pulsu(m) Plantae (2009-2012) de Leslie García, hace uso de la botánica, la fisiología, la filosofía, entre otros; por mencionar solo algunos desarrollos artísticos.

El proceso de la investigación del arte electrónico y sus artistas contribuye a entender el área para establecer parámetros que contribuyan a la creación de un posible programa educativo en arte electrónico, así como analizar si las condiciones actuales en universidades públicas mexicanas lo hacen posible. El proceso de la investigación fue explorar y describir para luego generar perspectivas teóricas, con cuyo acopio de datos se obtuvieron las perspectivas y puntos de vista de los participantes; características propias de la investigación cualitativa. Como estrategia de investigación se utilizó la entrevista directa por 
diversos medios, tales como, teléfono, correo electrónico, vídeo conferencia y en persona con artistas, profesores y responsables de eventos de arte digital; la visita directa a centros y eventos dedicados parcial o completamente al arte digital, tales como Laboratorio Arte Alameda, Aniversario de la revista Leonardo en México, asistencia al International Symposium on Electronic Art (ISEA2017 Manizales, ISEA2018 Durban), Ars Electronica Center Linz, entre otros.

Como parte de esta investigación, se estudiaron las características del público al cual va dirigido el arte electrónico mexicano y el contexto desde es producido. De acuerdo al Consejo Nacional de Evaluación de la Política de DesarroIlo Social (Coneval), en 2016 México presentaba 53.4 millones habitantes con diversos grados de pobreza y 9.4 millones en pobreza extrema, es decir, 62.8 millones de mexicanos de un total de 119.5 millones de habitantes. Lo anterior impacta la parcela del acceso a la tecnología, donde gran parte de la población mexicana no cuenta, por ejemplo, con una computadora personal: "La proporción de hogares que disponen de computadora [es de] 45.4\% en 2017" (INEGI 2018)2. En consecuencia, si el acceso a la tecnología es difícil, esto incide en que no existan suficientes artistas, ni suficientes espacios equipados para la creación y la experimentación.

Un aspecto importante que brinda diversos matices al tema del arte electrónico es la reutilización y el reciclaje. Se observó que algunos artistas mexicanos, tales como: Leslie García, Arcángel Constantini, Gilberto Esparza, entre otros, recurrieron a los tiraderos de basura tecnológica, a los materiales de bajo costo y al software libre como medio para desarrollar obra artística:

- Arma Sonora Telemática ${ }^{3}$ (2012) de Leslie García / Colectivo Astrovandalistas. Es una instalación sonora: “...encontramos una estructura metálica de $4 \times 6$ mts [sic] ubicada frente al Campo Marte, un campo militar que encierra historias de un pasado represivo que se ha ido olvidando poco a poco. Intervenimos la estructura con 64 tubos galvanizados conectados a un motor. Este mecanismo es activado a distancia cada vez que alguien escribe \#BANGCampoMarte en Twitter".

- Ánima / 3:00 am (s/f) de Arcángel Constantini: es un desarrollo en Internet donde los objetos son hilados por un discurso existencial basado en la teoría del artista del bucle conceptual o loop contínuo. La obra está compuesta por una retícula de 25 módulos, en cada uno se activa un vídeo del mercado de usado de Santa Martha Acatitla en 
la Ciudad de México; el audio fue realizado por el artista sonoro Manrico Montero ${ }^{5}$ y surge del sonido original del propio mercado.

- Parásitos urbanos ${ }^{6}$ (2006) de Gilberto Esparza: “...el proyecto contempla la creación de varias especies de parásitos robots que habitan en determinados lugares de la ciudad, y que son híbridos de desechos tecnológicos de distintas clases y materiales diversos. Los parásitos se insertan en el contexto urbano e intervienen el paisaje cotidiano con su presencia y a través de emisiones sonoras".

Hay que destacar que la reutilización de objetos usados o el reciclaje en la elaboración de un proyecto artístico, no es característica exclusiva del arte digital mexicano, el teórico del arte Nicolas Bourriaud destacó el mercado de pulgas como forma dominante de los noventa en el arte; de acuerdo a este autor, el mercado se volvió de uso recurrente en las prácticas artísticas porque: representa una forma colectiva de aglomeración caótica creciente que no depende de un único autor porque fue formado por innumerables individuos que de alguna forma reorganizaron la producción del pasado. Por lo que, de acuerdo al autor, en los mercados de usado se materializan relaciones humanas que tienden a desaparecer con la industrialización del comercio y las ventas por Internet (Bourriaud 2004, 30); sin embargo el países como Brasil y México donde hay producción destacada de arte electrónico, la reutilización y el reciclado son también una necesidad.

\section{PRODUCCIÓN ARTÍSTICA ELECTRÓNICA EN ENTORNOS AUSTEROS}

El desarrollo de proyectos de arte electrónico y digital en jóvenes contribuye al interés en el trabajo artístico colectivo y en la toma de conciencia de la identidad cultural. Es el caso del Laboratorio de arte digital del Colegio de Ciencias y Humanidades de la Universidad Nacional Autónoma de México (UNAM) a cargo del maestro y artista visual Alberto Barrios López y se encuentra funcionando desde el año 2015. En este espacio colaboran jóvenes bachilleres de entre quince y dieciocho años, en una modalidad autónoma (la materia no es obligatoria). En el laboratorio se les brinda capacitación gratuita de software libre, para el desarrollo de proyectos artísticos interactivos de forma autónoma o colectiva ${ }^{7}$. El $\mathrm{CCH}$ Vallejo en la Ciudad de México, se encuentra en la Delegación Gustavo A. Madero, que tiene el mayor número de perso- 
nas en pobreza extrema (junto con las delegaciones Ecatepec, Iztapalapa y Nezahualcoyótl, las cuales rodean geográficamente la Delegación Gustavo A. Madero) (CONEVAL 2013). Esta zona tiene un profundo simbolismo cultural nacional, ya que ahí se han descubierto asentamientos humanos de antiguas civilizaciones, las cuales datan del año 1500 a.C ${ }^{8}$. Lo anterior es trascendente porque se vincula a varios de los proyectos generados por los alumnos de dicho laboratorio, quienes año con año realizan ofrendas para conmemorar la tradición prehispánica del Día de muertos.

Es el caso de la obra Ofrenda digital 2017 realizada por un equipo de diez alumnos $^{9}$, inspirada por el fuerte sismo de 7.1 grados vivido el 19 de septiembre de 2017 a las 13:14 horas, justo cuando los alumnos se encontraban en clase. Fue debido a la fuerte impresión que experimentaron, que deciden canalizar la experiencia artísticamente: "[el temblor]... nos recordó que somos parte de un gran organismo vivo, como lo narra el mito de Tlalcihuat/ ${ }^{10}$ : La Mujer-Tierra", para ello los alumnos emplearon computadoras y el idioma Náhuatl en la representación de tres elementos aztecas: Tlalcihuatl (Mujer-tierra), Tonalpohualli (calendario sagrado de los aztecas), Quetzalcóatl (o Serpiente emplumada, dios de la cultura mesoamericana). Dispusieron los elementos en tres distintos desarrollos, que fueron llevados a cabo con animaciones coloridas, a través de la técnica de Video mapping, la elaboración de un holograma y la Serpiente emplumada desplazándose por 12 computadoras.

Otro desarrollo artístico interesante es el titulado Ataraxia, llevado a cabo por estudiantes de la Licenciatura en Arte y Comunicación Digitales de la Universi-

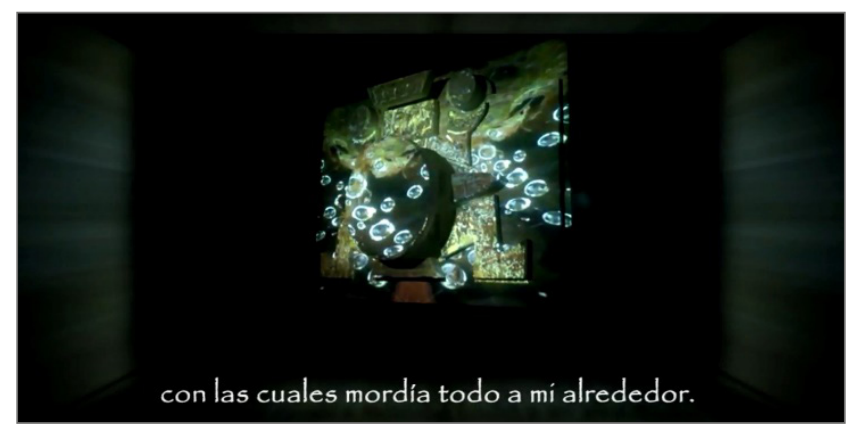

Ofrenda digital 2017, Laboratorio de arte digital CCH Vallejo, Imagen: https://www.youtube.com/watch?v=KtfFzfGZHtc\&t=17s con autorización del Profesor Alberto Barrios.

dad Autónoma Metropolitana unidad Lerma, quienes conformaron el Colectivo 

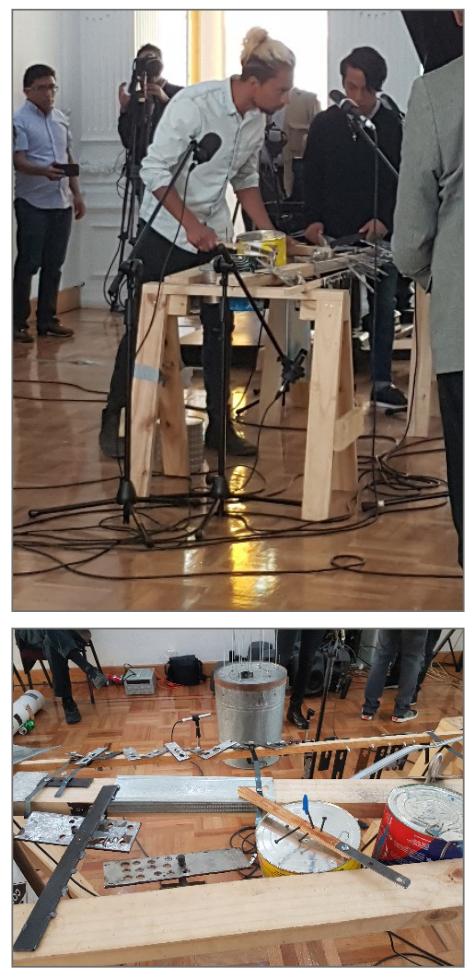

Ataraxia (2018), Edmar Olivares Soria y el Colectivo rékTwV_\{ $\left.{ }^{*}\right\}$.debug(). Centro Cultural Casa del Tiempo, Ciudad de México. Imágenes: Cynthia Villagómez.

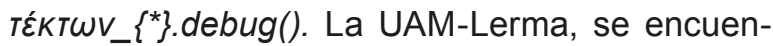
tra en Lerma de Villada en el Estado de México, la unidad es creada en 2009 y se encuentra en un municipio homónimo cuya actividad económica principal es la agricultura, en general es una zona con un índice de pobreza significativo ${ }^{11}$. Es importante mencionar, que la presentación del desarrollo artístico fue en 2018, un momento en que los alumnos trabajaban en aulas de lámina, pues el edificio de la universidad se terminó de construir apenas en enero de 2020 (García Garibay 2019).

La presentación de Ataraxia fue en el festejo del 50 Aniversario de la revista Leonardo ${ }^{12}$ en la Ciudad de México el 19 de abril de 2018. El profesor y artista sonoro Edmar Olivares Soria y el Colec-

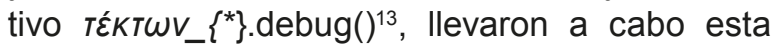
obra descrita como: electroacústica, multicanal para multipercusiones ensambladas, electrónica y vídeo.

En palabras de Edmar Olivares, el proyecto es una exploración sonora del concepto de la resonancia y el espacio, a partir del empalmado. Se utilizaron como percusiones latas de aluminio (de chiles y otras conservas), clavos, pedazos de madera y metal. Al respecto Edmar Olivares comentó que cada una de las piezas había sido cortada de modo distinto, algunas fueron perforadas para explorar sonidos diferentes, por ejemplo, sonidos armónicos. Es decir, los instrumentos fueron diseñados para obtener de ellos determinadas resonancias (crearon una cítara con una carcasa o caja exterior de un ordenador PC y unas cuerdas). La idea era crear a partir de materiales industriales y reciclados nuevos instrumentos capaces de producir sonidos diferentes. Olivares hizo énfasis en que la exploración principal fue con el espacio físico, es decir, la colocación de los sonidos en el espacio, ya que de acuerdo al timbre de cada sonido producido eligieron su posición espacial con el propósito de poder distinguir todas las capas de sonido producidas. ${ }^{14}$ 


\section{CONCLUSIONES}

Históricamente el arte ha reflejado el espíritu de la época a la que pertenece, en la actualidad estamos en la Era de la tecnología y la información, por lo que no es extraño que el arte adopte estos medios como recursos de expresión. Por otra parte, las hondas diferencias sociales entre los distintos continentes del orbe han generado diversas formas artísticas dentro del arte electrónico, las cuales se traducen en pequeños retratos de las realidades diametralmente opuestas que se viven en cada región. Mientras las economías del primer mundo tienen espacios destinados al arte con el uso de ciencia y tecnología de primer nivel, como SymbioticA en Australia, o FutureLab en Ars Electronica Center en Austria, los países en vías de desarrollo experimentan en pequeños talleres como Cráter Invertido en la Ciudad de México o Platohedro en Medellín, Colombia; lo anterior es parte del amplio espectro que abarca el panorama económico y social global, donde la noción de diversidad será la esencia para el entendimiento de las distintas expresiones artísticas.

El papel de la educación universitaria dentro de este escenario, es el de evitar el atraso, anteponiendo los factores sociales y económicos que intervienen en las instituciones de educación superior como agentes de cambio, así como en construir el andamiaje de mejores programas educativos que respondan a las necesidades de este siglo, donde el arte ocupa un lugar coyuntural para sanar el tejido social, sobre todo en países con rezagos importantes en aspectos tales como calidad de vida y acceso a la tecnología y a la información.

Muchos son los alcances que se pueden vislumbrar a través de la educación en el Arte electrónico, tales como un acceso igualitario a la ciencia y la tecnología, no solo para artistas, sino también para ciudadanos que deseen un mejor futuro y un mejor país.

\section{Referencias bibliografícas}

Bourriaud, Nicolas. (2000) 2004. Postproducción. Traducción de Silvio Mattoni. Buenos Aires: Adriana Hidalgo

CONEVAL (Consejo Nacional de Evaluación de la Política de Desarrollo Social). 2013."Pobreza urbana y de las zonas metropolitanas en México". Equipo técnico, Gonzalo Hernández Licona et al.; revisión técnica María del Rosario Cárdenas Elizalde et al. www.coneval.org. $\mathrm{mx} /$ Informes/Pobreza/Pobreza\%20urbana/Pobreza_urbana_y_de_las_zonas_metropolitanas_en_Mexico.pdf 
— . 2016. "Medición de la pobreza: Pobreza a nivel municipio 2010 y 2015". https://www. coneval.org.mx/Medicion/Paginas/Pobreza-municipal.aspx

García Garibay, José Mariano. 2019. "La unidad Lerma de la UAM, consolidada en el valle de Toluca”. Entrevista con el rector de la Universidad Autónoma Metropolitana de México, nota prensa 19 sept. http://www.ler.uam.mx/work/banner/Entrevista_de_UAM_Radio_al_ Dr_Mariano.pdf

INEGI (Instituto Nacional de Estadística y Geografía, México). 2018. "Encuesta nacional sobre disponibilidad y uso de tecnologías de la información en los hogares (ENDUTIH)”. www. inegi.org.mx/programas/dutih/2018/

Lieser, Wolf. 2010. Arte digital: Nuevos caminos en el arte. Con la colaboración de Tilman Baumgärtel et al.; traducción al español, Katinka Rosés Becker. Potsdam: H.F. Ullmann

Programa delegacional de desarrollo urbano de Gustavo A. Madero. 1997. DOF (Diario Oficial de la Federación, México), 12 mayo. http://dof.gob.mx/nota_detalle.php?codigo=4878849\&fecha $=12 / 05 / 1997$

Universidad de Guanajuato. 2016. "Guía para la modificación de programas educativos de licenciatura y técnico superior universitario". https://ugto.mx/images/guiamodeloe/guia-modificacion-pe-licenciatura-tsu-universidad-guanajuato.pdf

— . 2018. "Políticas y lineamientos generales de racionalidad, austeridad y disciplina presupuestal de la Universidad de Guanajuato para el ejercicio fiscal del año 2019". https://www. ugto.mx/images/pdf/politicas-lineamientos-generales-racionalidad-austeridad-disciplina-presupuestal-2019.pdf

Villagómez Oviedo, Cynthia Patricia. 2017. "Análisis de procesos de producción artística digital en México: Artistas digitales mexicanos y su obra”. Tesis Univ. Politècnica de València

\section{Notas}

${ }^{1}$ Es el caso de los egresados de la Licenciatura en Arte Digital de la Universidad de Guanajuato, de acuerdo a estudios de egresados de la institución.

2 Por otra parte "En México hay 71.3 millones de usuarios de Internet, que representan el 63.9 por ciento de la población de seis años o más" (INEGI 2018, 2).

${ }^{3}$ Arma Sonora Telemática (2012). Electrónica y código, Rodrigo Frenk \& Leslie Garcia. Less Null_Void, http://lessnullvoid.cc/content/2012/08/arma-sonora-telematica/

${ }^{4}$ Entrevistas realizadas por la autora publicadas en la cuenta de Vimeo de la autora: https:// vimeo.com/user29545447. Anima. http://www.unosunosyunosceros.com/anima/3am/index. htm

${ }^{5}$ Manrico Montero Calzadíaz, Ciudad de México (1973-2018). http://manricomontero.com/index. php/project/

${ }^{6}$ Parásitos Urbanos (2009). http://www.parasitosurbanos.com/parasitos/proyecto.html

${ }^{7}$ Entrevista de Cynthia Villagómez al Mtro. Alberto Barrios López, 19 de mayo de 2018. También expresó que el inicio del Laboratorio Digital se debió a que llegaron a la UNAM computadoras $\mathrm{PC}$ de última generación, con procesador Core 5 con 8 Gigas de RAM, además ya contaban con Internet de alta velocidad y decidieron aprovechar. Alberto Barrios -junto con el Lic. Héctor Tinajero- es fundador del laboratorio que dio inicio en Atzcapotzalco. En el Laboratorio utilizan el programa alemán V4, que integra a una comunidad muy grande, 
de ahí se inspiran para realizar sus actividades y de un libro llamado Prototyping Interfaces. El programa del curso está en: Laboratorio de Arte Digital. http://l-ad.blogspot.mx/

8 “...en la zona del Arbolillo, Ticomán y Zacatenco [...] Sin embargo en la zona llamada El Arbolillo, sitio que estuvo muy próximo al lago, se encontraron restos de una pequeña comunidad agrícola y enterramientos de huesos teñidos de rojo. Esta característica perdura en la cultura de Zacatenco, que florecía en la misma zona entre el año 100 a.C. y 100 d.C.".(Programa delegacional de desarrollo urbano de Gustavo A. Madero 1997).

${ }^{9}$ Daniela Fragoso, Genesis Garay, Daniela Meza, Pamela Mimila, Lizeth Noriega, Claudia Pérez, Diana Priego, Samantha Rodríguez, Diana Saldaña.

10 Tlalcihuatl Video mapping (2017). UNAM CCH Vallejo-Laboratorio de Arte Digital; profesor, Alberto Barrios López; traducción y Voz al náhuatl, Hortencia Vargas Dominguez; música orig. Euclides Rodríguez Valencia. Vídeo de Youtube, 4:55. https://www.youtube.com/wat$c h ? v=K t f F z f G Z H t c \& t=17 \mathrm{~s}$

${ }^{11}$ En 2016 porcentaje de $47.9 \%$ de personas en situación de pobreza y $6.1 \%$ en pobreza extrema. Documento en Excel: Medición de la pobreza Estados Unidos Mexicanos (Coneval 2016).

12 Prestigiosa publicación del Instituto de Tecnología de Massachusetts fue fundada por Frank Malina, publicación de la Sociedad Internacional de las Artes, las Ciencias y la Tecnología (ISAST).

${ }^{13}$ Integrado por Abiram Bello Gomeztagle, Alexa Amanda Bonilla Ybarra, Angélica Lara Batallar, José Carlos Camacho Carrasco, Josué Javier Martínez Alcántara, Ottmar Grover Ricalde Cardeño, Sergio Axayácatl Villagómez Cerda y Sussan Andrea Robledo Noriega.

${ }^{14}$ Entrevista de Cynthia Villagómez al Mtro. Edmar Olivares Soria después del concierto ofrecido en el Centro Cultural Casa del Tiempo en la Ciudad de México con motivo del 50 Aniversario de la Revista Leonardo.

(Artículo recibido: 16-03-20; aceptado: 08-05-20) 